\title{
Computed tomography coronary angiography is the way forward for evaluation of children with Kawasaki disease
}

Advanced Pediatrics Center, Post Graduate Institute of Medical Education and Research (PGIMER), Chandigarh, INDIA-160012

*Email: drmsinghal@yahoo.com http://dx.doi.org/

10.21542/gcsp.2017.28

Received: 2 August 2017 Accepted: 8 November 2017 (C) 2017 The Author(s), licensee Magdi Yacoub Institute. This is an open access article distributed under the terms of the Creative Commons Attribution license CC BY-4.0, which permits unrestricted use, distribution and reproduction in any medium, provided the original work is properly cited.

Manphool Singhal*, Pankaj Gupta, Surjit Singh, Niranjan Khandelwal

\begin{abstract}
Kawasaki disease (KD) is an acute idiopathic vasculitis affecting infants and children. Coronary artery abnormalities and myocarditis are the major cardiovascular complications of KD. Coronary artery abnormalities develop in 15-25\% of untreated KD. Two-dimensional transthoracic echocardiography has hitherto been considered the modality of choice for evaluation of children with KD. There are, however, several limitations inherent to echocardiography - including limited evaluation of distal vessels, left circumflex artery and poor acoustic window in growing children. Catheter angiography is the gold standard for evaluation of coronary artery abnormalities in older children and adults; however it also has inherent limitations - including complications related to its invasive nature, higher radiation exposure, and inability to evaluate intramural abnormalities. Thus serial invasive coronary angiography studies are not feasible in children. There have been major advances in computed tomography (CT) coronary imaging so that it is now possible to delineate the coronary artery anatomy with higher temporal resolution and motion-free images at all heart rates with acceptable radiation exposure. There is, however, a paucity of literature with regard to the use of this technique in children with KD. In this review, we discuss the application of computed tomography coronary angiography (CTCA) in children with KD with special reference to strategies aimed at reducing the effective radiation dose.
\end{abstract}




\section{INTRODUCTION}

$\mathrm{KD}$ is a vasculitic disorder in children with a predilection for coronary arteries. Without appropriate treatment, up to $25 \%$ children with KD can develop coronary artery abnormalities (CAA). With early diagnosis and prompt therapy the complications can be reduced to $3-5 \%$. Two-dimensional echocardiography is the imaging modality of choice for assessment of coronary arteries during the acute stage and on followup $^{1}$. Echocardiography, however, has its own inherent limitations. It is highly operator dependent, requires experience and may not be able to delineate distal segments of coronary arteries and left circumflex artery. Further, as the child grows, evaluation of coronary arteries becomes difficult because of a limited acoustic window ${ }^{1,2}$. Other imaging techniques can supplement echocardiography. However, there are no clear-cut recommendations or guidelines on their usage in children with $\mathrm{KD}$. Catheter angiography is an option but it is invasive, cannot be repeated at frequent intervals, requires sedation and is associated with high radiation exposure. It may also fail to detect intramural changes in coronary arteries ${ }^{3}$. Magnetic resonance (MR) coronary angiography is technically difficult and time consuming and few centres have the requisite expertise in performing the procedure in young children ${ }^{1}$. With significant advancements in $\mathrm{CT}$ technology including the availability of increased detector rows and advanced software, CTCA is expected to allow a comprehensive non-invasive evaluation including luminal changes, intramural changes and plaque morphology objectively along the entire course of coronary arteries at reasonably low radiation dose ${ }^{4}$. Thus CTCA is an alternative imaging modality for non-invasive diagnosis and follow-up of children with KD. Accordingly, its incorporation into the management algorithm of KD should be considered.

\section{CORONARY ARTERY LESIONS IN KD}

With early diagnosis and appropriate management, there has been a dramatic reduction in occurrence of CAA in children with $\mathrm{KD}$. However, approximately $3-5 \%$ of treated children still go on to develop CAA ${ }^{5,6}$. These CAA can evolve into stenotic lesions or occlusions. CAA may decrease in size during the convalescence phase ${ }^{4}$. However, remodelling of CAA occurs in only half of these patients over the first 2 years of follow$u^{6,7}$. All of these children may develop significant residual disease and complications in adulthood. This serves as the basis for long term cardiovascular follow-up of patients with KD. Coronary stenosis may be segmental or localized. Segmental stenosis refers to neovascularisation and recanalization in a previously occluded segment ${ }^{8}$. This complication is encountered in $15 \%$ patients and involves the right coronary artery most frequently. Localised stenosis of greater than $75 \%$ develops in $5-12 \%$ patients in the second decade following disease onset ${ }^{9}$. It has a predilection for proximal trunk of left anterior descending.

\section{IMAGING IN FOLLOW UP OF KD}

CAAs in $\mathrm{KD}$ require long-term follow-up. The aim is to determine the progression of the CAA and monitor development of thrombotic and steno-occlusive complications. The American Heart Association (AHA) proposes the application of interval echocardiography, stress echocardiography, and invasive or non-invasive coronary angiography in follow up of patients with $\mathrm{KD}^{1}$.

Echocardiography is the imaging technique of choice for follow up ${ }^{6}$. Limitations of echocardiography include operator dependence and poor delineation of distal segments 


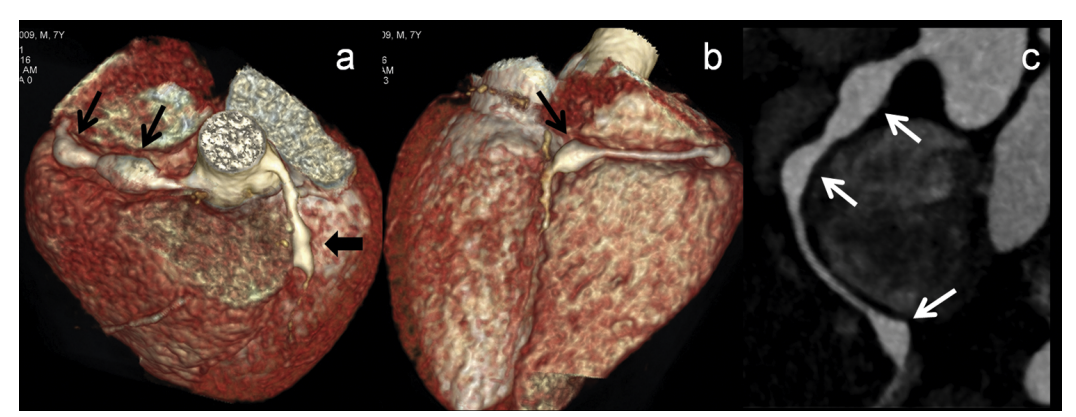

Figure 1. $(a-c)$ : CT Coronary angiography (CTCA) images (a \& b - volume rendered images-VRT) and (c) curved maximum intensity projection (MIP) images of a 7 year male child in acute phase show two fusiform aneurysms in proximal right coronary artery (RCA) and one in distal RCA at its bifurcation into its posterior descending and postero-lateral branches (thin arrows). Note fusiform aneurysm in mid left anterior descending artery (LAD) as well (a-thick arrow). 2D-transthoracic echocardiography could detect one aneurysm in proximal RCA and proximal LAD and missed two aneurysms in RCA.

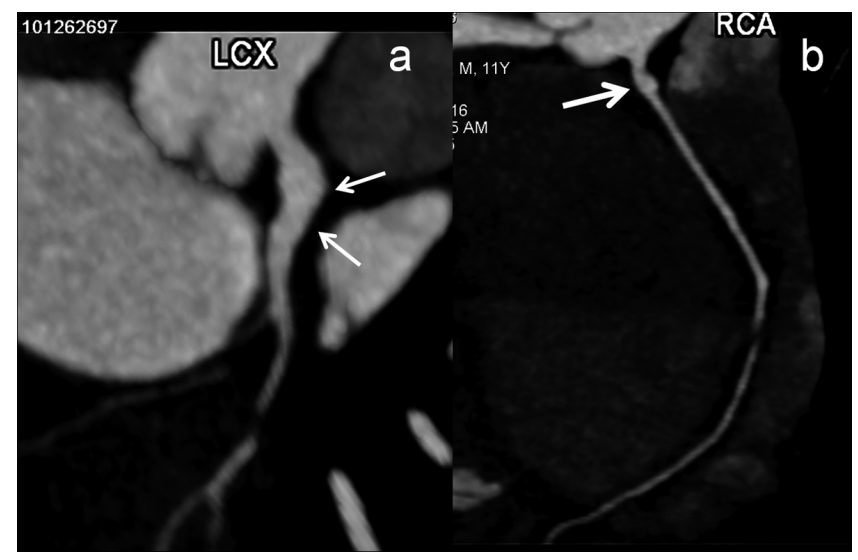

Figure 2. (a-b): CTCA curved maximum intensity projection (MIP) images of a 11 year male child in convalescent phase ( 5 years after diagnosis) show fusiform aneurysmal dilation ( $a$ - thin arrows) of proximal left circumflex artery (LCX) and saccular aneurysm of proximal RCA (thick arrow). Left circumflex artery is usually not well evaluated on 2D-transthoracic echocardiography.

of the coronary arteries and left circumflex artery ${ }^{1}$ (Figures 1 and 2). Besides, it has poor sensitivity in demonstrating intra-luminal thrombus or arterial stenosis ${ }^{1}$. Furthermore, as the child grows, assessment of coronary arteries becomes more difficult. Nevertheless, the preliminary examination performed in the acute phase serves as a baseline for longitudinal follow-up.

Invasive catheter angiography is considered the gold standard for evaluation of coronary arteries. However, it is an invasive procedure and is associated with significant complications. Its routine use in serial follow up of children with KD is also limited by the associated radiation hazard. Typical dose in diagnostic catheter angiography is 5-7 mSv ${ }^{10}$. Though, catheter angiography is exquisitely sensitive to intraluminal changes, it does not specifically detect intramural changes. Post-mortem studies of patients with KD in whom CAA were shown to regress with catheter angiography revealed intimal proliferation and fibrosis not detected on angiogram ${ }^{11}$. As mural changes are an important component of cardiovascular complications in KD, an imaging modality that is capable of evaluating the lumen as well as the wall of coronary arteries is necessary. 


\section{CT CORONARY ANGIOGRAPHY}

\section{Background and evolution}

With the early generations of CT scanners, sensitivity for grading native coronary disease was low. However with advent of 64-slice CT, gantry rotation time was reduced to $330 \mathrm{~ms}$ leading to marked improvement in temporal resolution for cardiac electrocardiography (ECG)-gated imaging ${ }^{12}$. This translated to improved image quality and high sensitivity and specificity in the evaluation of all coronary artery branches and segments. Increase in the number of acquired slices to 128, 256 and 320 and resultant increase in z-axis coverage has further improved the temporal resolution ${ }^{13}$. With 320-slice CT, cranio-caudal coverage of $16 \mathrm{~cm}$ allows demonstration of the entire coronary arteries in a single heartbeat ${ }^{14}$. With dual-source CT (DSCT), the temporal resolution is improved to $73 \mathrm{~ms}$. This reduces the need to control the heart rate during the scan. Studies comparing the diagnostic performance of DSCT and single-source CT in the evaluation of coronary arteries of a wide range of patient subsets (including those with atrial fibrillation) demonstrated high diagnostic accuracy for DSCT ${ }^{14}$. This is particularly vital in children who cannot be administered large doses of beta-blockers.

\section{Rationale for use of CTCA in KD}

Changes in coronary circulation in KD affect the lumen as well as the wall. In this context, CTCA not only has the capability to depict changes in luminal calibre, it can also evaluate intramural changes including plaque characterisation. The current generation of CT scanners have a spatial and temporal resolution that allows the evaluation of all coronary artery branches and segments with exquisite detail. Thus, CTCA is an ideal non-invasive imaging modality for evaluation of CAA in KD.

\section{Challenges: radiation issues}

The prime concern in the use of CTCA in children is the radiation hazard. Although CT constitutes only $15 \%$ of all radiological investigations, it entails high radiation dose and accounts for up to $70 \%$ radiation dose from diagnostic radiological procedures ${ }^{15}$. This becomes highly relevant in view of the recent reports of radiation induced malignancy. It is estimated that a radiation exposure of $10 \mathrm{mSv}$ will result in development of malignancy in 1 in 2000 population exposed ${ }^{16}$. In a study by Brenner and Hall, $1.5-2 \%$ of all cancers in the USA were attributed to radiation exposure from CT scans ${ }^{15}$. Thus an appropriate balance between radiation dose and image quality must be reached. Concerns over escalating radiation doses have led to various strategies being adopted for dose reduction in CTCA. Fortunately, all of the latest generation scanners (at least 256 detector rows) provide better image quality and lower patient dose than earlier generation scanners. Of all the dose-saving strategies in CTCA including child-size bowtie filter, body size-adapted protocol including low tube voltage techniques; ECG-controlled and attenuation-based tube current modulations, and prospectively ECG-triggered sequential scanning, the last approach is the most critical and is discussed below in detail.

\section{PROSPECTIVE ECG TRIGGERING}

Prospective ECG triggering is based on the premise that CT data is acquired only during the particular cardiac phase by stimulating the $X$-ray tube only when signalled by the ECG signal. In rest of the R-R interval, the $X$-ray tube current is switched off or markedly lowered. A lowering of effective dose up to $90 \%$ compared to retrospective ECG-gating has been reported in studies using single-source 64 -slice or DSCT ${ }^{17-19}$. This comes at a diagnostic value similar to that of CTCA with retrospective ECG-gating. With application 
of prospective ECG triggering, a radiation dose comparable to catheter angiography has been reported. The condition for successful application of this technique is a low and regular heart rate. Thus, heart rate control with pharmacological measures is required for prospective gating. However, with the advent of DSCT, the heart rate control for prospective gating has been rendered less strict. Misalignment due to acquisition of images in more than one heartbeat is another potential limitation of prospective gating ${ }^{17}$. This is partly overcome by the latest 320-slice CT, which allows the entire heart volume to be covered in a single heartbeat.

A study by Duan et al. in 19 children with $\mathrm{KD}$ reported the mean $\mathrm{CT}$ doses of prospective ECG-triggered CTCA as low as $0.4 \mathrm{mSv}^{19}$. Thus it can be seen that recent CTCA technology has led to acceptable CT radiation dose. In a study assessing the doses from various pediatric protocols in infants using a phantom, effective doses of $1.49 \pm 0.10$ and $4.66 \pm 0.40 \mathrm{mSv}$ for non-gated and retrospectively gated scans, respectively was reported in a 64 -slice CT scanner ${ }^{20}$. In a 256-slice CT, effective doses were $2.15 \pm 0.15$, $6.87 \pm 0.56$ and $1.12 \pm 0.11 \mathrm{mSv}$ for non-gated, retrospectively gated and prospectively gated scans, respectively ${ }^{20}$. The authors concluded that prospectively gated CTCA protocol allows good quality images with relatively low dose. Prospectively gated CTCA protocol in fact had better image quality than the retrospectively gated scan with $83.70 \%$ dose reduction ${ }^{20}$.

Huang et al. conducted a study to assess the diagnostic performance of prospectively gated 256-slice CTCA in infants ${ }^{21}$. They reported that diagnostic quality images were possible in all cases with diagnostic accuracy up to $95.9 \%$.

Duan et al. investigated the role of prospective ECG-triggered dual-source CT coronary angiography in 19 infants and children with KD and coronary artery aneurysms ${ }^{19}$. They found that the image quality and inter-observer agreement were excellent. The mean effective dose was $0.36 \pm 0.06 \mathrm{mSv}$.

In a study by Kim et al., five children below the age of 2 years, a radiation dose of only $0.6 \pm 0.5 \mathrm{mSv}$ (range, $0.2-1.3 \mathrm{mSv}$ ) was reported using prospective ECG-triggered sequential CT scanning ${ }^{22}$. Our own experience with prospective ECG-triggering is in line with the results of studies that support its routine use in pediatric CTCA 23,24.

Using the dose saving strategies discussed above, doses less than 1 mSv have been reported in the literature ${ }^{25}$. This can be achieved with a combination of strategies including prospective ECG triggering. With such a low radiation dose, widespread application of CTCA in children in general and those with KD in particular should become routine.

\section{CT CORONARY ANGIOGRAPHY: COMPARISON WITH ECHOCARDIOGRAPHY}

All the segments of the main coronary arteries may be involved in KD, although the lesions are most often proximal. It is often not appreciated that echocardiography has only a limited role in evaluation of middle and distal coronary arteries and left circumflex artery ${ }^{26}$. CTCA provides an ideal method for evaluation of all the segments of the coronary artery (Figures 3 and 4 ).

In a study of 24 children with KD, the efficacy of echocardiography was compared with CTCA in evaluation of $\mathrm{CAA}^{26}$. Echocardiography failed to detect 8 small aneurysms located in mid and distal segments [LAD2 $(n=1), \operatorname{LCX}(n=2), \operatorname{RCA} 2 / 3(n=4)$ and D1 $(n=1)]$. CTCA also detected a stenosis of LAD1 that was missed on echocardiography.

Chu et al. reported failure of echocardiography to detect the distal lesions in a study comprising 8 children with KD ${ }^{27}$. Echocardiography missed 3 of the 12 aneurysms located in $\operatorname{RCA}_{3}(n=1)$ and LAD1 $(n=2)$. 


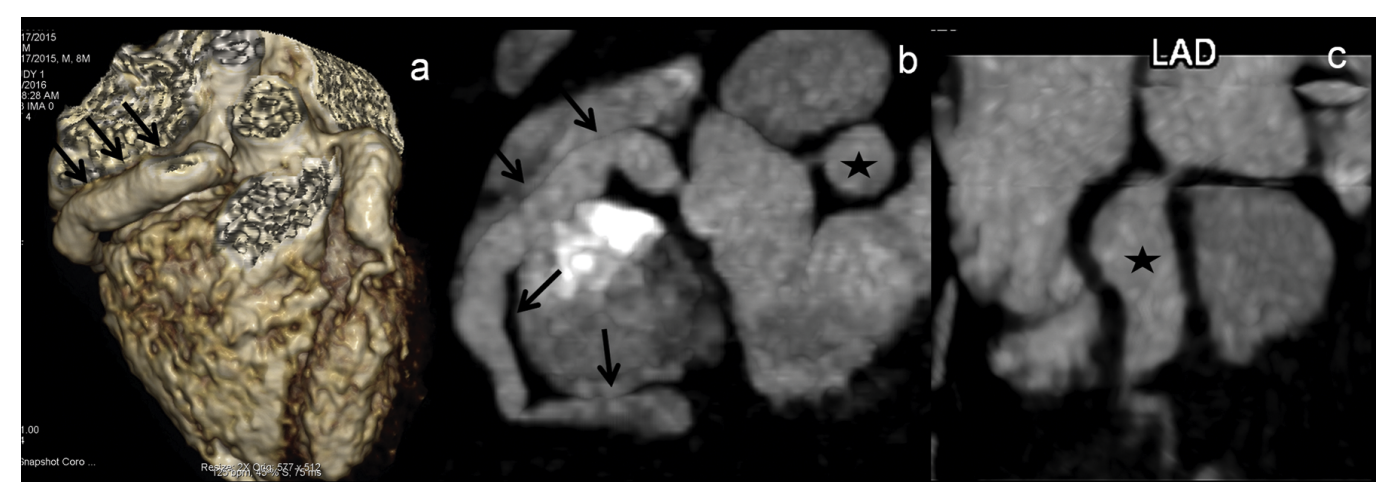

Figure 3. (a-c): CTCA VRT (a) and curved (MIP) images (b \& c) of an 8 year male child in acute phase show aneurysmal dilation of entire RCA ( $a$ \& - thin arrows) and a giant aneurysm in proximal LAD (b \& c - asterisk). 2D-transthoracic echocardiography detected aneurysm in both the arteries; however it failed to delineate the extent of involvement of RCA.

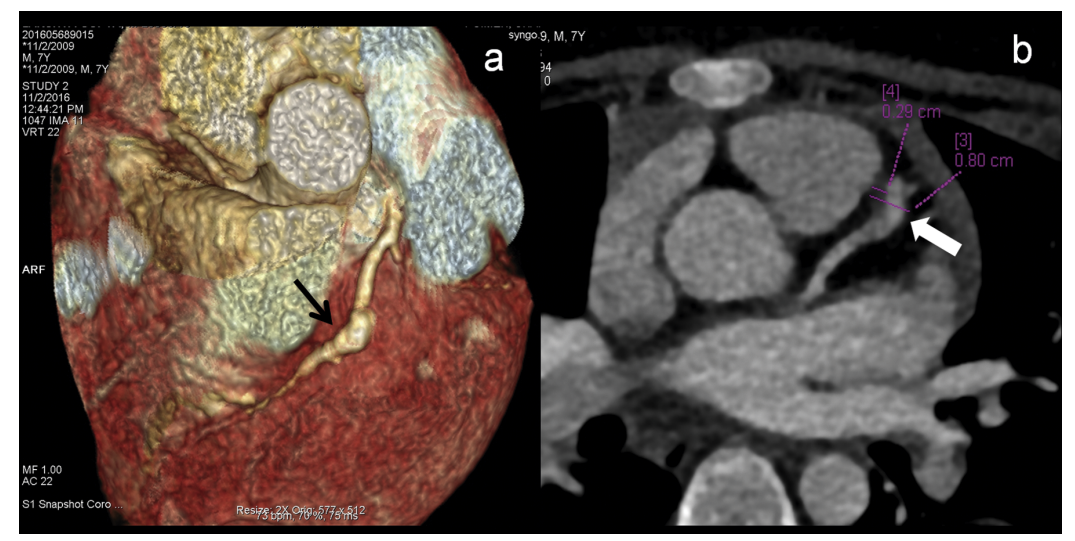

Figure 4. (a-b): CTCA VRT (a) axial (b) images of a 7 year male child in convalescent phase (4 years after diagnosis) show fusiform aneurysmal dilation (a - thin arrows) of proximal LAD; (b) shows a giant aneurysm with eccentric thrombus along the anterior wall (thick arrow). 2D-transthoracic echocardiography though could detect aneurysm but thrombus was missed.

In the study by Xing et al. 4 aneurysms were missed in the LCA $(n=3)$ and LAD $(n=1)$ on 2D-echocardiography. In addition, stenosis and calcification were missed in the LCA $(n=3)^{28}$.

Peng et al. reported the comparison of CTCA and 2D-echocardiography in 12 boys with KD. Echocardiography missed 8 of the 30 aneurysms detected by CTCA ${ }^{29}$. These lesions were location in the $\operatorname{LAD}(n=2), \operatorname{LCX}(n=1)$, and RCA $(n=5)$. In addition, echocardiography missed calcification and stenotic lesions.

In the study by Duan et al., echocardiography failed to detect 7 aneurysms ${ }^{19}$. In all the reported studies, there was a good correlation between echocardiography and CTCA for the size of the detected aneurysms.

\section{CTCA: COMPARISON WITH MR CORONARY ANGIOGRAPHY (MRCA)}

MRCA has also been evaluated as one of the non-invasive imaging modalities in patients with KD. In a usual clinical setting, one has to choose between MRCA and CTCA as the best imaging modality. Studies in a small cohort of patients with KD, having only aneurysms, have reported good agreement between MRCA and CCA ${ }^{30,31}$. However, others have reported lower diagnostic performance of MRCA ${ }^{32,33}$. This can be attributed to lower 


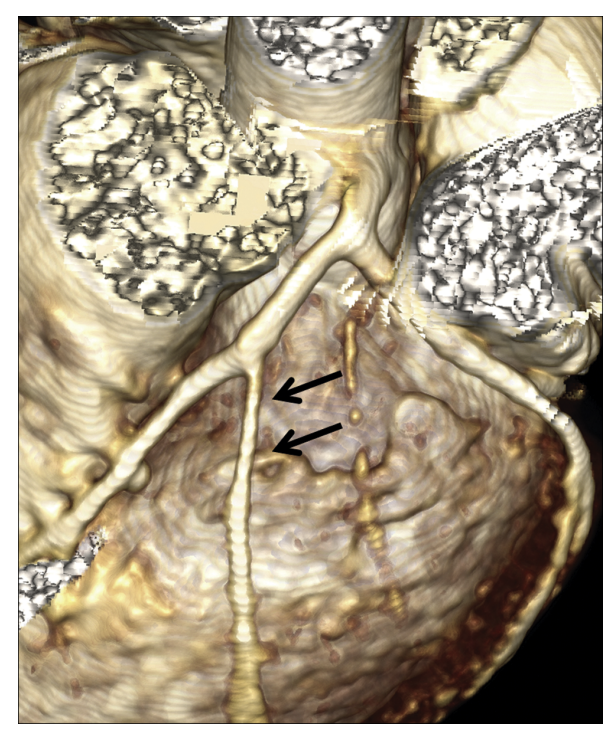

Figure 5. CTCA VRT 8 year female child in convalescent phase (5 years after diagnosis) show long segment stenosis of diagonal branch of LAD (arrows). 2D-transthoracic echocardiography has limitation to detect coronary artery abnormalities of coronary artery branches.

spatial and temporal resolution and poorer image quality compared to CTCA 34 . The longer scan time of MRCA in young children is particularly detrimental to the technical success rate of MRCA ${ }^{35}$. Studies on patients with steno-occlusive lesions reported a greater accuracy of CTCA over MRCA (Figure 5). Situations in which MRCA is preferred over CTCA include evaluation of coronary arteries with heavy intra-mural calcifications (leading to blooming artifact on $\mathrm{CT}$ ) and assessment of myocardial perfusion, viability, and fibrosis ${ }^{36}$.

\section{CTCA: COMPARISON WITH CATHETER ANGIOGRAPHY}

Catheter angiography is an invasive procedure and requires hospital admission for duration dependant on institutional protocols. There is a definite risk of minor and major complications. Although, it has the highest spatial resolution that although evaluation of the coronary artery lumen with a high degree of accuracy, it fails in evaluation of the coronary artery wall. CTCA scores over catheter angiography in evaluation of intraluminal thrombus, wall thickening and calcification. There are only a few studies prospectively comparing the performance of catheter angiography and CTCA in children with KD but the advantages of CTCA are clearly demonstrated by its utilisation in other conditions. In a study by Tsujii et al., the accuracy of CTCA and catheter angiography in measurement of coronary artery aneurysms was compared ${ }^{37}$. Twenty-two children with coronary artery lesions were retrospectively evaluated. There was excellent agreement between CTCA and catheter angiography in measurement of aneurysms.

\section{CONCLUSION}

CTCA is a robust imaging modality in evaluation of children with KD. With studies reporting $\mathrm{CT}$ doses less than $1 \mathrm{mSv}$, it is expected to be used more frequently both in the acute and chronic phases of the disease. In near future, CTCA may be incorporated in the management algorithm of children with KD. 


\section{ACKNOWLEDGEMENTS}

None

\section{FUNDING}

This research received no grant from any funding agency in the public, commercial or notfor-profit sectors.

\section{DISCLOSURES}

The authors declare that there are no conflicts of interest.

\section{ABBREVIATIONS}

$\begin{array}{ll}\text { CTCA } & \text { Computed tomography coronary angiography } \\ \text { CAA } & \text { Coronary arterial abnormalities } \\ \text { CTDI vol } & \text { CT dose index } \\ \text { DLP } & \text { Dose length product } \\ \text { DSCT } & \text { Dual source computed tomography } \\ \text { ECG } & \text { Electrocardiogram } \\ \text { KD } & \text { Kawasaki disease } \\ \text { MR } & \text { Magnetic resonance }\end{array}$

\section{REFERENCES}

[1] McCrindle BW, Rowley AH, Newburger JW, Burns JC, Bolger AF, Gewitz M, Baker AL, Jackson MA, Takahashi M, Shah PB, Kobayashi T, Wu MH, Saji T, Pahl E, E. American Heart Association Rheumatic Fever, Y. Kawasaki Disease Committee of the Council on Cardiovascular Disease in the, C. Council on, N. Stroke, S. Council on Cardiovascular, Anesthesia, E. Council on and Prevention. Diagnosis, treatment, and long-term management of Kawasaki disease: A scientific statement for health professionals from the American heart association. Circulation. 2017;135(17):e927-e999.

[2] Sokmen G, Tuncer C, Sokmen A, Suner A. Clinical and angiographic features of large left main coronary artery aneurysms. Int J Cardiol. 2008;123:79-83.

[3] Sato Y, Kato M, Inoue F, et al. Detection of coronary artery aneurysms, stenosis and occlusions by multislice spiral computed tomography in adolescents with Kawasaki disease. Circ J. 2003;67:427-30.

[4] Suzuki A, Kamiya T, Tsuda E, Tsukano S. Natural history of coronary artery lesions in Kawasaki disease. Prog Pediatr Cardiol. 1997;6:211-8.

[5] Kato H, Sugimura T, Akagi T, et al. Long-term consequences of Kawasaki disease. A 10- to 21-year followup study of 594 patients. Circulation. 1996;94:1379-85.

[6] Suzuki A, Kamiya T, Arakaki Y, Kinoshita Y, Kimura K. Fate of coronary arterial aneurysms in Kawasaki disease. Am J Cardiol. 1994;74:822-4.

[7] Suzuki A, Kamiya T, Ono Y, Kinoshita Y, Kawamura S, Kimura K. Clinical significance of morphologic classification of coronary arterial segmental stenosis due to Kawasaki disease. Am J Cardiol. 1993;71:1169-73.

[8] Suzuki A, Kamiya T, Ono Y, Kohata T, Kimura K, Takamiya M. Follow-up study of coronary artery lesions due to Kawasaki disease by serial selective coronary arteriography in 200 patients. Heart Vessels. 1987:3:159-65.

[9] Takahashi K, Oharaseki T, Naoe S. Pathological study of postcoronary arteritis in adolescents and young adults: With reference to the relationship between sequelae of Kawasaki disease and atherosclerosis. PediatrCardiol. 2001;22:138-42.

[10] Pugliese F, Mollet NR, Runza G, et al. Diagnostic accuracy of non-invasive 64-slice CT coronary angiography in patients with stable angina pectoris. EurRadiol. 2006;16:575-82.

[11] Mizuno N, Funabashi N, Imada M, et al. Utility of 256-slice cone beam tomography for real fourdimensional volumetric analysis without electrocardiogram gated acquisition. Int J Cardiol. 2007;120:262-7.

[12] deGraaf FR, Schuijf JD, van Velzen JE, et al. Diagnostic accuracy of 320-row multidetector computed tomography coronary angiography in the non-invasive evaluation of significant coronary artery disease. Eur Heart J. 2010;35:1908-15.

[13] Pasricha SS, Nandurkar D, Seneviratne SK, et al. Image quality of coronary 320-MDCT in patients with atrial fibrillation: Initial experience. AJR Am J Roentgenol. 2009;193:1514-21. 
[14] Committee to assess health risks from exposure to low levels of ionizing radiation, nuclear and radiation studies board, division on earth and life studies, National Research Council of the National Academies.

[15] Brenner DJ, Hall EJ. Computed tomography-An increasing source of radiation exposure. N Engl I Med. 2007:357:2277-84.

[16] Sun Z, Ng KH. Multislice CT angiography in cardiac imaging-Part III: Radiation risk and dose reduction. Singapore Med J. 2010;51:374-80.

[17] Husmann L, Valenta I, Gaemperli O, et al. Feasibility of low-dose coronary CT angiography: First experience with prospective ECG-gating. Eur Heart J. 2008;29:191-7.

[18] Shuman WP, Branch KR, May JM, et al. Prospective versus retrospective ECG gating for 64-detector CT of the coronary arteries: Comparison of image quality and patient radiation dose. Radiology. 2008;248:431-7.

[19] Duan Y, Wang X, Cheng Z, Wu D, Wu L. Application of prospective ECG-triggered dual-source CT coronary angiography for infants and children with coronary artery aneurysms due to Kawasaki disease. Br J Radiol. 2012;85:e1190-7.

[20] Lee Y-W, Yang C-C, Mok GSP, Wu T-H. Infant cardiac CT angiography with 64-slice and 256-slice CT: Comparison of radiation dose and image quality using a pediatric phantom. PLOS ONE. 2012;7:e49609.

[21] Huang B, Li J, Law MWM, Zhang J, Shen Y, Khong PL. Radiation dose and cancer risk in retrospectively and prospectively ECG-gated coronary angiography using 64-slice multidetector CT. Br J Radiol. 2010;83:152-8.

[22] Kim JW, Goo HW. Coronary artery abnormalities in Kawasaki disease: Comparison between CT and MR coronary. Acta Radiol. 2013;54:156-63.

[23] Singhal M, Singh S, Gupta P, Sharma A, Khandelwal N, Burn JC. Computed tomography coronary angiography in evaluation of children with Kawasaki disease. Curr Probl Diagn Radiol. 2017 (ahead of print). doi: 10.1067/j.cpradiol.2017.09.013.

[24] Tsuda E, Singhal M. Role of imaging studies in Kawasaki disease. Int J Rheum Dis. 2017. doi: 10.1111/1756-185X.13210. [Epub ahead of print].

[25] Gosling O, Loader R, Venables P, et al. A comparison of radiation doses between state-of-the-art multislice CT coronary angiography with iterative reconstruction, multislice CT coronary angiography with standard filtered back-projection and invasive diagnostic coronary angiography. Heart. 2010;96:922-6.

[26] Yu Y, Sun K, Wang R, et al. Comparison study of echocardiography and dual-source CT in diagnosis of coronary artery aneurysm due to Kawasaki disease: Coronary artery disease. Echocardiography. 2011;28:1025-1034.

[27] Chu WC, Mok GC, Lam WW, Yam MC, Sung RY. Assessment of coronary artery aneurysms in pediatric patients with Kawasaki disease by multidetector row CT angiography: Feasibility and comparison with 2D echocardiography. Pediatr Radiol. 2006;36:1148-53.

[28] Xing Y, Wang H, Yu X, Chen R, Hou Y. Assessment of coronary artery lesions in children with Kawasaki disease: Evaluation of MSCT in comparison with 2-D echocardiography. Pediatr Radiol. 2009;39:1209-15.

[29] Peng Y, Zeng J, Du Z, et al. Usefulness of 64-slice MDCT for follow-up of young children with coronary artery aneurysm due to Kawasaki disease: Initial experience. Eur J Radiol. 2009;69:500-9.

[30] Greil GF, Seeger A, Miller S, et al. Coronary magnetic resonance angiography and vessel wall imaging in children with Kawasaki disease. Pediatr Radiol. 2007;37:666-73.

[31] Takemura A, Suzuki A, Inaba R, et al. Utility of coronary MR angiography in children with Kawasaki disease. AJR Am J Roentgenol. 2007;188:W534-9.

[32] Chuijf JD, Bax JJ, Shaw LJ, et al. Meta-analysis of comparative diagnostic performance of magnetic resonance imaging and multislice computed tomography for noninvasive coronary angiography. Am Heart J. 2006;151:404-11.

[33] Bluemke DA, Achenbach S, Budoff M, et al. Noninvasive coronary artery imaging: Magnetic resonance angiography and multidetector computed tomography angiography: A scientific statement from the American heart association committee on cardiovascular imaging and intervention of the council on cardiovascular radiology and intervention, and the councils on clinical cardiology and cardiovascular disease in the young. Circulation. 2008;118:86-606.

[34] Gupta P, Gulati GS, Kothari SS. Kawasaki disease: A rare case of diffuse coronary involvement. Pediatr Cardiol. 2012;33:1218-9.

[35] Deetjen A, Mollmann S, Conradi G, et al. Use of automatic exposure control in multislice computed tomography of the coronaries: Comparison of 16-slice and 64-slice scanner data with conventional coronary angiography. Heart. 2007;93:1040-3.

[36] Zhang S, Levin DC, Halpern EJ, et al. Accuracy of MDCT in assessing the degree of stenosis caused by calcified coronary artery plaques. AJR Am J Roentgenol. 2008;191:1676-83.

[37] Tsujii N, Tsuda E, Kanzaki S, Kurosaki K. Measurements of coronary artery aneurysms due to Kawasaki disease by dual-source computed tomography (DSCT). Pediatr Cardiol. 2016;37:442-7. 